\section{DGI-021 COST-EFFICACY ANALYSIS OF ABIRATERONE FOR THE TREATMENT OF HORMONE-REFRACTORY METASTATIC PROSTATE CANCER PATIENTS}

doi:10.1136/ejhpharm-2013-000276.287

A Alcobia, A Soares. Hospital Garcia de Orta, Pharmacy, Almada, Portugal

Background In combination with prednisone or prednisolone, abiraterone is indicated for the treatment of patients with hormonerefractory metastatic prostate cancer (mHRPC) previously treated with a docetaxel-containing regimen. Abiraterone was evaluated in a phase 3, randomised, double-blind, placebo-controlled study.

Purpose To evaluate the cost-efficacy of abiraterone for the treatment of patients with mHRPC previously treated with a docetaxelcontaining regimen, using best supportive care as a comparator.

Materials and Methods Abiraterone efficacy and safety data were sourced directly from the above-mentioned phase 3 study. Two different efficacy parameters were considered: overall survival (OS) and progression free survival (PFS). The costs of the therapeutic options were calculated based on the direct cost of the drugs and the treatment duration described in the study. This study was conducted from an institutional perspective - the hospital perspective. Results In the phase III trial considered, the median OS was 14.8 months with abiraterone and 10.9 months with placebo. The median PFS was 10.2 months in the abiraterone group and 6.6 months in the placebo group. Median treatment duration was eight months for abiraterone and four months for placebo. The marginal efficacy for abiraterone is 3.9 months for OS and 3.6 months for PFS. Considering OS as efficacy parameter, the incremental costefficacy ratio (ICER) calculated for the two treatments is $€ 89.848$. When PFS is considered, the ICER calculated is $€ 97.336$.

Conclusions Based on this analysis, the ICERs calculated for abiraterone are too high for it to be considered a cost-effective option in the treatment of mHRPC when compared with mitoxantrone, in patients patients previously treated with a docetaxel-containing regimen.

No conflict of interest.

\section{DGI-022 COST-EFFICACY ANALYSIS OF CABAZITAXEL FOR THE TREATMENT OF HORMONE-REFRACTORY METASTATIC PROSTATE CANCER PATIENTS}

doi:10.1136/ejhpharm-2013-000276.288

A Alcobia, A Soares. Hospital Garcia de Orta, Pharmacy, Almada, Portugal

Background In combination with prednisone or prednisolone, cabazitaxel is indicated for the treatment of patients with hormonerefractory metastatic prostate cancer (mHRPC) previously treated with a docetaxel-containing regimen. Cabazitaxel was evaluated versus mitoxantrone in an open-label randomised phase III trial, the TROPIC study.

Purpose To evaluate the cost-efficacy of cabazitaxel for the treatment of patients with mHRPC previously treated with a docetaxelcontaining regimen, using mitoxantrone as a comparator.

Materials and Methods Cabazitaxel and mitoxantrone efficacy and safety data were sourced directly from the TROPIC trial. Two different efficacy parameters were considered: overall survival (OS) and progression free survival (PFS). The costs of the two therapeutic options were calculated based on the direct cost of the drugs, treatment duration and the probability of using granulocyte colonystimulating factors (filgrastim). This study was conducted from an institutional perspective - the hospital perspective.

Results In the TROPIC trial, the median OS was 15.1 months with cabazitaxel and 12.7 months with mitoxantrone, and median PFS was 2.8 months in the cabazitaxel group and 1.4 months in the mitoxantrone group. Median number of treatment cycles was six for cabazitaxel and four for mitoxantrone. The most frequent clinically significant grade $3 / 4$ adverse events were neutropenia (cabazitaxel $(82 \%)$ vs. mitoxantrone $(58 \%))$. The marginal efficacy of cabazitaxel vs. mitoxantrone is 2.4 months for OS and 1.4 months for PFS. Considering OS as efficacy parameter, the incremental costefficacy ratio (ICER) calculated for the two treatments is $€ 147.389$. When PFS is considered, the ICER calculated is $€ 248.871$.

Conclusions Based on this analysis, the ICERs calculated for cabazitaxel are too high for it to be considered a cost-effective option in the treatment of mHRPC, when compared with mitoxantrone, in patients previously treated with a docetaxel-containing regimen.

No conflict of interest.

\section{DGI-023 DESCRIPTION OF OMALIZUMAB USE FOR THE TREATMENT OF ASTHMA AFTER FOUR YEARS OF EXPERIENCE}

doi:10.1136/ejhpharm-2013-000276.289

L Serrano De Lucas, A Bustinza Txertudi, B Baza Martinez, S Sautua Larreategui, A De Basagoiti Gorordo, I Bilbao Meseguer, A Belaustegui Foronda, Z Baskaran Kaltzakorta, J Hernandez Goicoechea, E Rodriguez España. Hospital Universitario Cruces, Pharmacy, Barakaldo, Spain

Background Omalizumab's labelled indication is the treatment of IgE-mediated asthma. It has been used in our hospital since 2008. In 2011 it became necessary to develop a protocol that clarified patient selection and criteria for withholding treatment.

Purpose To describe the patients treated with omalizumab, focusing on whether they match our protocol's use criteria or not.

Materials and Methods All patients treated with omalizumab for asthma in our hospital were included. Data were obtained in October 2012 from electronic clinical records: treatment period, patient smoker or not, other medicines for asthma, basal IgE, adherence to treatment, omalizumab dosage and hospitalizations and emergency department visits before and after treatment.

Our omalizumab use protocol states these patient selection criteria: uncontrolled severe asthma with previous optimised therapy, basal IgE $>76 \mathrm{IU} / \mathrm{mL}$ and at least three emergency department visits or one hospitalisation in the previous year. Treatment withholding criteria are: evaluation after 16 weeks and stop if treatment shows no benefit.

Two different pharmacists examined each patient's information to establish if treatment was being effective and whether the hospital's protocol was being followed.

Results 31 patients were studied, 7 children and 24 adults. Treatment was stopped in 9 patients, due to lack of efficacy in 8 of them and to adverse effects in the other (diarrhoea, fever and skin reaction). Previous treatments included montelukast or theophylline in 19 patients (61\%). Basal IgE was below $76 \mathrm{IU} / \mathrm{mL}$ in one patient. Median duration of treatment was 637 days (72-1624). Regarding patients' adherence to treatment, $23 \%$ of patients missed a dose, $13 \%$ missed two and $6 \%$ missed three or more. 13 patients had had no pre-treatment hospitalizations or emergency department visits.

Treatment was evaluated as effective in 14 of the 22 patients who continued receiving omalizumab (64\%).

Conclusions Our patients still need to be selected better. Protocol compliance is lower than desirable.

No conflict of interest.

DGI-024 DEVELOPMENT OF A GUIDE FOR ADMINISTERING ANTIVIRAL DRUGS BY GASTROSTOMY OR NASOGASTRIC TUBE

doi:10.1136/ejhpharm-2013-000276.290

J Arenas Villafranca, M Nieto Guindo, C Lopez Martin, R Romero Dominguez, ME Blanco Rivas, M Garrido Siles. 'Empresa Pública Hospital Costa del Sol, Farmacia Y Nutrición, Marbella, Spain 
Background The number of patients infected by HIV and hepatitis has increased over the years. Some of them have swallowing difficulties that require the placement of nasogastric or gastrostomy tubes. These chronic treatments need high compliance rates to avoid antiviral drug resistance and, eventually, treatment failure.

Purpose To review the existing antiviral drugs literature and develop administration recommendations for patients with swallowing problems.

Materials and Methods Formulations and recommendations were obtained directly from the manufacturers, or by a PubMed search and a search on the Micromedex database, when information was not available. A guide published by SENPE with physicochemical and formulation properties of drugs was also checked.

Results Table 1 shows the results. Extensive administration recommendations were found during literature searches but are not included in the present abstract. There was no information about the administration of adefovir, maraviroc or saquinavir through gastrostomy or nasogastric tube.

Conclusions Treatment compliance is key to ensuring the success of chronic antiviral treatments and it is important to consider special situations, such as swallowing problems. This guide for nasogastric or enteral administration helps clinicians to choose the most appropriate treatment. Further research is needed to determine specific bioavailability data.

Abstact DGI-024 Table 1 Antiviral Drug Formulations and Administration

\begin{tabular}{|c|c|c|}
\hline Drug & $\begin{array}{l}\text { Solution available } \\
\text { (mg/ml solution) }\end{array}$ & $\begin{array}{l}\text { Can be crushed/ } \\
\text { sprinkled }\end{array}$ \\
\hline abacavir & 20 & Yes \\
\hline didanosine & $2 \mathrm{~g} / \mathrm{ml}$ solution powder & Use tablets, not capsules \\
\hline emtricitabine & 10 & Discouraged \\
\hline lamivudine & 10,5 & Yes \\
\hline stavudine & 1 & Yes \\
\hline tenofovir & & Yes \\
\hline zidovudine & 50 & Discouraged \\
\hline efavirenz & $30^{*}$ & Use capsules \\
\hline etravirine & & Yes \\
\hline nevirapine & 10 & Discouraged \\
\hline $\begin{array}{l}\text { atazanavir } \\
\text { darunavir }\end{array}$ & $50 \mathrm{mg} / 1.5 \mathrm{~g}$ solution powder * & $\begin{array}{l}\text { Discouraged } \\
\text { Yes }\end{array}$ \\
\hline fosamprenavir & 50 & \\
\hline indinavir & & Discouraged \\
\hline $\begin{array}{l}\text { lopinavir/ritonavir } \\
\text { nelfinavir }\end{array}$ & $80 / 20$ & $\begin{array}{l}\text { Discouraged } \\
\text { Yes }\end{array}$ \\
\hline ritonavir & 80 & Discouraged \\
\hline tipranavir & 100 & \\
\hline raltegravir & & Yes \\
\hline abacavir/lamivudine & ** & \\
\hline abacavir/lamivudine/zidovudine & ** & \\
\hline zidovudine/lamivudine & $* *$ & Yes \\
\hline tenofovir/emtricitabine & & Yes \\
\hline tenofovir/emtricitabine/efavirenz & & Discouraged \\
\hline boceprevir & & Discouraged \\
\hline telaprevir & & Discouraged \\
\hline ribavirin & 40 & Discouraged \\
\hline entecavir & $0.05^{*}$ & \\
\hline telbivudine & $20^{*}$ & Discouraged \\
\hline
\end{tabular}

* Not in Spain

** Individual drugs available in solution

No conflict of interest.

\section{DGI-025 DEVELOPMENT OF A PROTOCOL FOR THE TREATMENT OF VITAMIN D DEFICIENCY/INSUFFICIENCY IN ADULTS}

doi:10.1136/ejhpharm-2013-000276.291

'M Kieran, 'D O'Sullivan, ${ }^{2} \mathrm{~J}$ Brady, ${ }^{3} \mathrm{~S}$ McQuaid, ' $\mathrm{C}$ Meegan. ${ }^{1}$ Mater Misericordiae University Hospital, Pharmacy Department, Dublin, Ireland (Rep.); '2Mater Misericordiae University Hospital, Biochemistry Department, Dublin, Ireland (Rep.); ${ }^{3}$ Mater Misericordiae University Hospital, Department of Endocrinology, Dublin, Ireland (Rep.)
Background Recent medical research has highlighted that vitamin $\mathrm{D}$ deficiency/insufficiency is a significant public health problem. A UK study found that more than $50 \%$ of the adult population had insufficiency and $16 \%$ had deficiency. [1] Low vitamin D levels have been linked to rickets, malignancies, cardiovascular disease, type 2 diabetes and some autoimmune diseases. [1] Therefore, appropriate management of Vitamin D deficiency/insufficiency is essential.

This increased awareness among prescribers of treating vitamin $\mathrm{D}$ deficiency was apparent in the Mater Misericordiae University Hospital (MMUH):

- Medicines Information enquiries regarding treatment of vitamin $\mathrm{D}$ deficiency had increased.

- Biochemistry assay numbers for vitamin D (25-hydroxyvitamin D) had increased.

- Requests to the endocrinology service for guidance on the treatment of vitamin D deficiency had increased.

MMUH clinicians were experiencing difficulty treating patients with vitamin $\mathrm{D}$ deficiency/insufficiency as:

- There were no definitive guidelines for the treatment of vitamin D deficiency/insufficiency.

- There is no licenced preparation containing cholecalciferol or ergocalciferol as a single drug formulation in Ireland.

Guidance for MMUH clinicians was therefore necessary.

Purpose To develop a protocol for the treatment of vitamin D deficiency/insufficiency.

Materials and Methods Review of treatment algorithms for treatment of vitamin D deficiency/insufficiency in the literature.

Compilation of vitamin D products currently available in Ireland.

Liaison with MMUH clinical staff to ensure production of a protocol that is applicable to all disciplines.

Results A treatment algorithm was prepared detailing two specific guidelines for the treatment of:

- Vitamin D deficiency (serum 25-hydroxy-vitamin D $<25$ $\mathrm{nmol} / \mathrm{L}$ )

- Vitamin D insufficiency (serum 25-hydroxy-vitamin D 25-50 $\mathrm{nmol} / \mathrm{L}$ )

The protocol recommends vitamin $\mathrm{D}$ preparations, including one unlicensed preparation, which are available in the MMUH and accessible in the community.

The protocol also recognises the limitation of giving guidance on treating a condition that may be affected by numerous clinical scenarios or that may require input from specialist physicians. Where applicable, consultation with the relevant medical team(s) is recommended.

Conclusions MMUH patients diagnosed with vitamin D deficiency/insufficiency are treated in a standardised manner in accordance with available clinical evidence. The protocol ensures delays in treatment are minimised and physicians are aware of the particular considerations involved in the management of vitamin $\mathrm{D}$ deficiency/insufficiency.

No conflict of interest.

\section{DGI-026 DRUG USE IN PATIENTS WITH METASTATIC BREAST CANCER}

doi:10.1136/ejhpharm-2013-000276.292

A Madrid Paredes, R López Sepúlveda, E Puerta García, N Martínez Casanova, B Cancela Díez, MA Calleja Hernández. Virgen de las Nieves University Hospital, Pharmacy, Granada, Spain

Background The historic poor prognosis and survival of metastatic breast cancer $(\mathrm{MBC})$ patients has been improved in the last decades by the introduction of multimodal treatment.

Purpose To analyse the $\mathrm{MBC}$ population and describe the prescription profile used. 